\title{
A CLASS OF SECOND ORDER TANGENT SETS
}

\author{
S. S. Kutateladze
}

June 16, 2020

\begin{abstract}
Under consideration are the construction and properties of some special class of second other tangent sets on using the technique of nonstandard analysis.
\end{abstract}

Let $X$ be a real vector space. Assume that we are given some almost vector topology $\sigma$ with the zero neighborhood filter $\mathcal{N}_{\sigma}:=\sigma(0)$ as well as some almost vector topology $\tau$ with the filter $\mathcal{N}_{\tau}:=\tau(0)$.

Recall that every almost vector topology $\sigma$ on $X$ is characterizes by the two properties: Firstly, multiplication by each scalar is continuous; and, secondly, addition is jointly continuous. It is clear that $X$ admits an almost vector topology $\sigma$ such that $\sigma(0)$ coincides with a fixed filter $\mathcal{N}$ if and only if the monad $\mu(\mathcal{N})$ is an external vector space over the external field of standard scalars.

In the sequel, $\sigma$ will be a vector topology, unless stated otherwise explicitly. It is comfortable to work in the assumption of standard environment within Nelson internal set theory IST (see [1]). Recall that the monad $\mu(\mathcal{F})$ of a standard filter $\mathcal{F}$ is the external intersection of the standard elements of $\mathcal{F}$. As usual, introduce the infinite proximity that is associated with the appropriate uniformity in $X$, i.e. $x_{1} \approx{ }_{\sigma} x_{2} \leftrightarrow x_{1}-x_{2} \in \mu\left(\mathcal{N}_{\sigma}\right)$. Note that the monad $\mu_{\sigma}(x)$ of the neighborhood filter $\sigma(x)$ of the topology $\sigma$ is as follows: $\mu_{\sigma}(x):=x+\mu\left(\mathcal{N}_{\sigma}\right)$. Let $\approx$ stand for the infinite proximity on the reals $\mathbb{R}$.

Recall that if given are some subset $F$ of $X$ and some point $\bar{x}$ in $X$, then subdifferential calculus (see [2] ) deals in particular with the Hadamard, Clarke, and Bouligand cones

$$
\begin{aligned}
& \operatorname{Ha}(F, \bar{x}):=\bigcup_{\substack{U \in \sigma(\bar{x}) \\
\lambda>0}} \operatorname{int}_{\tau}\left(\bigcap_{\substack{x^{\prime} \in F \cap \mathcal{T}^{\prime} \\
0<\lambda^{\prime} \leq \lambda}} \frac{F-x^{\prime}}{\lambda^{\prime}}\right) ; \\
& \mathrm{Cl}(F, \bar{x}):=\bigcap_{V \in \mathcal{N}_{\tau}} \bigcup_{\substack{U \in \sigma(\bar{x}) \\
\lambda>0}} \bigcap_{\substack{x^{\prime} \in F \cap U \\
0<\lambda^{\prime} \leq \lambda}}\left(\frac{F-x^{\prime}}{\lambda^{\prime}}+V\right) ;
\end{aligned}
$$

Key words and phrases. second order tangent, Clarke cone, Nelson internal set theory. Accepted for publication in Siberian Mathematical Journal@2020 Pleiades Publ. Ltd. http://pleiades.online/ 


$$
\mathrm{Bo}(F, \bar{x}):=\bigcap_{\substack{U \in \sigma\left(x^{\prime}\right) \\ \lambda>0}} \operatorname{cl}_{\tau}\left(\bigcup_{\substack{x \in F \cap U \\ 0<\lambda^{\prime} \leq \lambda}} \frac{F-x^{\prime}}{\lambda^{\prime}}\right),
$$

where, as usual, $\sigma(\bar{x}):=\bar{x}+\mathcal{N}_{\sigma}$. If $h \in \operatorname{Ha}(F, \bar{x})$ then $F$ is often called epilipshitzian at $\bar{x}$ with respect to $h$. It is obvious that

$$
\mathrm{Ha}(F, \bar{x}) \subset \mathrm{Cl}(F, \bar{x}) \subset \mathrm{Bo}(F, \bar{x}) .
$$

Considering an extended real function $f: X \rightarrow \mathbb{R} \cup\{+\infty\}$, the author of [3] defined the second order upper subderivative at $\bar{x} \in X$ along directions $\bar{v}, \bar{v}_{1}$, and $\bar{v}_{2}$ as follows:

$$
f_{\bar{x}}^{(2)}\left(\bar{v}, \bar{v}_{1}, \bar{v}_{2}\right):=\limsup _{\substack{x, \alpha) \rightarrow \bar{x} \\ \lambda \downarrow 0, \mu \downarrow 0}} \inf \underset{\substack{v \rightarrow \bar{v}_{1} \\ v_{1} \rightarrow \bar{v}_{1} \\ v_{2} \rightarrow \bar{v}_{2}}}{ } O_{f}^{2}\left(x, \alpha, \lambda, \mu, v, v_{1}, v_{2}\right),
$$

where we use the limit construction that is attributed to Painlevé, Kuratowski, and Rockafellar (for instance, see [1, Section 5.3],[4], or [5]) while putting

$$
\begin{gathered}
O_{f}^{2}\left(x, \alpha, \lambda, \mu, v, v_{1}, v_{2}\right) \\
:=\lambda^{-1} \mu^{-1}\left(f\left(x+\lambda v_{1}+\mu v_{2}+\lambda \mu v\right)-f\left(x+\lambda v_{1}\right)-f\left(x+\mu v_{2}\right)+\alpha\right) .
\end{gathered}
$$

Here $(x, \alpha) \rightarrow \bar{x}$ stands for the convergence to $(\bar{x}, f(\bar{x}))$ in the induced topology of the epigraph of $f$. The article [6] contains some approach to the explicit description of the tangent sets that are determined by similar constructions. The description uses the tools of IST.

As we will see soon, it is convenient to slightly modify the above construction by inserting the multiplier 4 but retaining the previous notations:

$$
f_{\bar{x}}^{(2)}\left(\bar{v}, \bar{v}_{1}, \bar{v}_{2}\right):=\limsup _{\substack{(x, \alpha) \rightarrow \bar{x} \\ \lambda \downarrow 0, \mu \downarrow 0}} \inf \underset{\substack{v \rightarrow \bar{v}_{1} \\ v_{1} \rightarrow \bar{v}_{1} \\ v_{2} \rightarrow \bar{v}_{2}}}{ } O_{f}^{2}\left(x, \alpha, \lambda, \mu, v, v_{1}, v_{2}\right),
$$

where we now put

$$
\begin{gathered}
O_{f}^{2}\left(x, \alpha, \lambda, \mu, v, v_{1}, v_{2}\right) \\
:=\lambda^{-1} \mu^{-1}\left(f\left(x+\lambda v_{1}+\mu v_{2}+4 \lambda \mu v\right)-f\left(x+\lambda v_{1}\right)-f\left(x+\mu v_{2}\right)+\alpha\right) .
\end{gathered}
$$

Given $F \subset X$, denote the indicator function of $F$ by $\delta_{F}$; i. e., $\delta_{F}(x):=0$ at $x \in F$ and $\delta_{F}(x):=\infty$ at $x \notin F$. Introduce the set $\mathrm{Cl}^{(2)}(F, \bar{x})\left(v_{1}, v_{2}\right)$ as follows:

$$
v \in \mathrm{Cl}^{(2)}(F, \bar{x})\left(v_{1}, v_{2}\right) \leftrightarrow\left(v, v_{1}, v_{2}\right) \in \operatorname{dom}\left(\delta_{F}\right)_{\bar{x}}^{(2)} .
$$

Considering the case of a normed space with $\tau$ - the norm topology and $\sigma$ - the discrete topology, $\mathrm{Cl}^{(2)}(F, \bar{x})(v, v)$ coincides with $\mathrm{A}^{(2)}(F, \bar{x}, v)$ - the second order attainable direction set to $F$ at $(\bar{x}, v)$ provided that $\bar{x}+\operatorname{cnt}(v)$ lies in $F$. As usual, $\operatorname{cnt}(v):=\{\lambda v: \lambda>0, \lambda \approx 0\}$ is the conatus of $v$ (see [1, Subsection 5.1.2]). Recall (see, for instance, [7]) that

$$
\mathrm{A}^{(2)}(F, \bar{x}, v):=\left\{h \in X:\left(\forall \lambda_{n} \downarrow 0\right)\left(\exists h_{n} \rightarrow h\right) \bar{x}+\lambda_{n} v+\frac{1}{2} \lambda_{n}^{2} h_{n} \in F\right\} .
$$

To simplify bulky formulas we will assume that $f$ is continuous at $\bar{x} \in F$ with respect to the topology $\tau$ on $X$. 
1. The following holds:

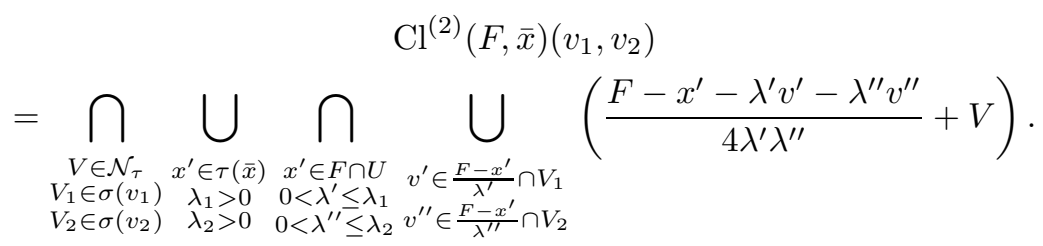

Proof. By transfer it suffices to check the case of standard parameters. Theorem 5.3.11 of [1] yields

$$
\begin{gathered}
v \in \mathrm{Cl}^{(2)}(F, \bar{x})\left(v_{1}, v_{2}\right) \\
\leftrightarrow\left(\forall x^{\prime} \approx_{\tau} \bar{x}, x^{\prime} \in F\right)\left(\forall \lambda^{\prime} \approx 0, \lambda^{\prime \prime} \approx 0, \lambda^{\prime}>0, \lambda^{\prime \prime}>0\right) \\
\left(\exists v_{1}^{\prime} \approx_{\sigma} v_{1}\right)\left(\exists v_{2}^{\prime} \approx_{\sigma} v_{2}\right)\left(\exists v^{\prime} \approx_{\sigma} v\right) \\
x^{\prime}+\lambda^{\prime} v_{1}^{\prime} \in F \wedge x^{\prime}+\lambda^{\prime \prime} v_{2}^{\prime} \in F \wedge x^{\prime}+\lambda^{\prime} v_{1}^{\prime}+\lambda^{\prime \prime} v_{2}^{\prime}+4 \lambda^{\prime} \lambda^{\prime \prime} v^{\prime} \in F .
\end{gathered}
$$

Denote the set on the right-hand side of the claim by $A$. Take $v \in \mathrm{Cl}^{(2)}(F, \bar{x})\left(v_{1}, v_{2}\right)$ and some standard standard neighborhoods $V \in \mathcal{N}_{\tau}, V_{1} \in \sigma\left(v_{1}\right)$, and $V_{2} \in$ $\sigma\left(v_{2}\right)$. If $\lambda_{1}$ and $\lambda_{2}$ are strictly positive infinitesimal while $U$ is an infinitesimal $\tau$-neighborhood of $\bar{x}$, i. e. $U \subset \mu_{\sigma}(\bar{x})$; then there are some $v_{1}^{\prime} \approx v_{1}, v_{2}^{\prime \prime} \approx_{\sigma} v_{2}$, and $v^{\prime} \approx_{\sigma} v$ such that $x^{\prime}+\lambda^{\prime} v_{1}^{\prime} \in F, x^{\prime}+\lambda^{\prime \prime} v_{2}^{\prime} \in F$, and $x^{\prime}+\lambda^{\prime} v_{1}^{\prime}+\lambda^{\prime \prime} v_{2}^{\prime}+4 \lambda^{\prime} \lambda^{\prime \prime} v^{\prime} \in F$ for all $x^{\prime} \in F \cap U, 0<\lambda^{\prime} \leq \lambda_{1}$, and $0<\lambda^{\prime \prime} \leq \lambda_{2}$. In other words, there exist $v_{1}^{\prime} \in\left(F-x^{\prime}\right) / \lambda^{\prime} \cap V_{1}, v_{2}^{\prime} \in\left(F-x^{\prime}\right) / \lambda^{\prime \prime} \cap V_{2}$, and $v^{\prime} \in v+V$ satisfying the needed properties. Since the parameters are standard, conclude that $v \in A$.

Assume now that $v \in A$. Take some standard neighborhoods $V \in \mathcal{N}_{\tau}, V_{1} \in \sigma\left(v_{1}\right)$ and $V_{2} \in \sigma\left(v_{2}\right)$ once again. By transfer there are $U \in \sigma(\bar{x}), \lambda_{1}>0$, and $\lambda_{2}>0$ such that $x^{\prime}+x^{\prime} v_{1}^{\prime}+\lambda^{\prime \prime} v_{2}^{\prime}+4 \lambda^{\prime} \lambda^{\prime \prime} v^{\prime} \in F$ for all $x^{\prime} \in F \cap U, 0<\lambda^{\prime} \leq \lambda_{1}$, and with some $v_{1}^{\prime} \in\left(F-x^{\prime}\right) / \lambda^{\prime} \cap V_{1}, v_{2}^{\prime} \in\left(F-x^{\prime}\right) / \lambda^{\prime \prime} \cap V_{2}$, and $v^{\prime} \in v+V$. Recalling that $x^{\prime} \in U$ if $x^{\prime} \approx \bar{x}$ and appealing to the properties of infinitesimals, we infer by idealization that $v \in \mathrm{Cl}^{(2)}(F, \bar{x})\left(v_{1}, v_{2}\right)$. The proof of Theorem 1 is complete.

Theorem 2. The following hold:

(1) If $\mathrm{Cl}^{(2)}(F, \bar{x})\left(v_{1}, v_{2}\right) \neq \varnothing$ then $v_{1}$ and $v_{2}$ belongs to the Clarke cone $\mathrm{Cl}(F, \bar{x})$.

(2) If $v_{1}, v_{2} \in \mathrm{Ha}(F, \bar{x})$ then $\mathrm{Cl}^{(2)}(F, \bar{x})\left(v_{1}, v_{2}\right)$ is a closed semigroup.

Proof. Claim (1) becomes obvious on recalling that in the standard environment we have

$$
h \in \mathrm{Cl}(F, \bar{x}) \leftrightarrow\left(\forall x^{\prime} \approx_{\sigma} \bar{x}, x^{\prime} \in F\right)\left(\forall \alpha^{\prime}>0, \alpha^{\prime} \approx 0\right)\left(\exists h^{\prime} \approx_{\tau} h\right) x^{\prime}+\alpha^{\prime} h^{\prime} \in F .
$$

Take $u_{1}, u_{2} \in \operatorname{Ha}(F, \bar{x})$. Without loss of generality, we will proceed in the standard environment. Therefore,

$$
\begin{aligned}
& v_{1} \in \operatorname{Ha}(F, \bar{x}) \leftrightarrow\left(\forall x^{\prime} \approx_{\sigma} \bar{x}, x^{\prime} \in F\right)(\forall \alpha>0, \alpha \approx 0)\left(\forall v_{1}^{\prime} \approx_{\tau} v_{1}\right) x^{\prime}+\alpha^{\prime} v_{1}^{\prime} \in F \\
& v_{2} \in \operatorname{Ha}(F, \bar{x}) \leftrightarrow\left(\forall x^{\prime} \approx_{\sigma} \bar{x}, x^{\prime} \in F\right)(\forall \alpha>0, \alpha \approx 0)\left(\forall v_{2}^{\prime} \approx_{\tau} v_{2}\right) x^{\prime}+\alpha^{\prime} v_{2}^{\prime} \in F .
\end{aligned}
$$


Assume now that $u_{1}, u_{2} \in \mathrm{Cl}^{(2)}(F, \bar{x})\left(v_{1}, v_{2}\right)$. By Theorem 1 we can write that

$$
\begin{gathered}
\left(\forall x^{\prime} \approx_{\sigma} \bar{x}, x^{\prime} \in F\right)\left(\forall \lambda^{\prime}>0, \lambda^{\prime} \approx 0\right)\left(\forall \lambda^{\prime \prime}>0, \lambda^{\prime \prime} \approx 0\right) \\
\left(\exists v_{1}^{\prime} \approx_{\tau} v_{1}\right)\left(\exists v_{2}^{\prime} \approx_{\tau} v_{2}\right)\left(\exists u^{\prime} \approx_{\tau} u_{1}\right) \\
x^{\prime \prime}:=x^{\prime}+\lambda^{\prime} v_{1}^{\prime}+\lambda^{\prime \prime} v_{2}^{\prime}+4 \lambda^{\prime} \lambda^{\prime \prime} u^{\prime} \in F .
\end{gathered}
$$

Using the properties of the vector topology $\sigma$ and its monad $\mathcal{N}_{\sigma}$, conclude that $x^{\prime \prime} \approx_{\sigma} \bar{x}$. Recalling Theorem 1 once again, we find $v_{1}^{\prime \prime} \approx_{\tau} v_{1} ; v_{2}^{\prime \prime} \approx v_{2}$, and $u^{\prime \prime} \approx u_{2}$ satisfying $x^{\prime \prime} \lambda^{\prime} v_{1}^{\prime \prime}+\lambda^{\prime \prime} v_{2}^{\prime \prime}+4 \lambda^{\prime} \lambda^{\prime \prime} u^{\prime \prime} \in F$. Put $v^{\prime}:=v_{1}^{\prime}+v_{2}^{\prime \prime}, v^{\prime \prime}:=v_{2}^{\prime}+v_{2}^{\prime \prime}$, and $u:=u^{\prime}+u^{\prime \prime}$. Undoubtedly, $v^{\prime} \approx_{\tau} v_{1}, v^{\prime \prime} \approx_{\tau} v_{2}$, and $u \approx_{\tau} u_{1}+u_{2}$. Furthermore, $x^{\prime}+\lambda^{\prime} v^{\prime} \in F$ and $x^{\prime}+\lambda^{\prime \prime} v^{\prime \prime} \in F$ since $v_{1}$ and, $v_{2}$ are hypertangents, i.e. elements of $\mathrm{Ha}(F, \bar{x})$. Moreover,

$$
\begin{gathered}
x^{\prime}+\lambda^{\prime} v^{\prime}+\lambda^{\prime \prime} v^{\prime \prime}+4 \lambda^{\prime} \lambda^{\prime \prime} u=x^{\prime}+\lambda^{\prime} v_{1}^{\prime}+\lambda^{\prime} v_{2}^{\prime}+\lambda^{\prime \prime} v_{2}^{\prime}+\lambda^{\prime \prime} v_{2}^{\prime \prime}+4 \lambda^{\prime} \lambda^{\prime \prime} u^{\prime}+4 \lambda^{\prime} \lambda^{\prime \prime} u^{\prime \prime} \\
=\left(x^{\prime}+\lambda^{\prime} v_{2}^{\prime}+\lambda^{\prime \prime} v_{2}^{\prime \prime}+4 \lambda^{\prime} \lambda^{\prime \prime} u^{\prime}\right)+\lambda^{\prime} v_{2}^{\prime}+\lambda^{\prime \prime} v_{2}^{\prime \prime}+4 \lambda^{\prime} \lambda^{\prime \prime} u^{\prime \prime} \\
=x^{\prime \prime}+\lambda^{\prime} v_{1}^{\prime}+\lambda^{\prime \prime} v_{2}^{\prime \prime}+4 \lambda^{\prime} \lambda^{\prime \prime} u^{\prime \prime} \in F .
\end{gathered}
$$

Consequently, $u_{1}+u_{2} \in \mathrm{Cl}^{(2)}(F, \bar{x})\left(v_{1}, v_{2}\right)$.

To prove closedness, take $u_{0} \in \mathrm{cl}_{\sigma} \mathrm{Cl}^{(2)}(F, \bar{x})\left(v_{1}, v_{2}\right)$ and some standard neighborhoods $V, V_{1}, V_{2} \in \mathcal{N}_{\tau}$ such that $V_{1}+V_{2} \subset V$. There is a standard vector $u \in \mathrm{Cl}^{(2)}(F, \bar{x})\left(v_{1}, v_{2}\right)$ satisfying $u-u_{0} \in V_{1}$. Moreover, using Theorem 1, we conclude that there are $x^{\prime} \approx_{\sigma} \bar{x}, x^{\prime} \in F, \lambda^{\prime} \approx 0, \lambda^{\prime}>0, \lambda^{\prime \prime} \approx 0$, and $\lambda^{\prime \prime}>0$ such that $u^{\prime} \in u+V_{2}, v^{\prime} \in v_{1}+W_{1}$, and $v^{\prime \prime} \in W_{2}+v_{2}$ for the previously given standard neighborhoods $W_{1}, W_{2} \in \mathcal{N}_{\tau}$ satisfying the containments $v^{\prime}+\lambda^{\prime} v^{\prime} \in F$, $v^{\prime}+\lambda^{\prime \prime} v^{\prime \prime} \in F$, and $x^{\prime}+\lambda^{\prime} v^{\prime}+\lambda^{\prime \prime} v^{\prime \prime}+4 \lambda^{\prime} \lambda^{\prime \prime} u^{\prime} \in F$. This implies easily that $u^{\prime} \in u+V_{2} \subset u_{0}+V_{1}+V_{2} \subset u_{0}+V$. By idealization we find $v^{\prime} \approx v_{1}, v^{\prime \prime} \approx_{\tau} v_{2}$ and $u_{0}^{\prime} \approx_{\tau} u_{0}$ such that $x^{\prime}+\lambda^{\prime} v^{\prime} \in F, x^{\prime}+\lambda^{\prime \prime} v^{\prime} \in F$, and $x^{\prime}+\lambda^{\prime} v^{\prime}+\lambda^{\prime \prime} v^{\prime \prime}+4 \lambda^{\prime} \lambda^{\prime \prime} u_{0}^{\prime} \in F$. This means that $u_{0} \in \mathrm{Cl}^{(2)}(F, \bar{x})\left(v_{1}, v_{2}\right)$.

Remark. Theorems 1 and 2 can be generalized to the case of the epiderivatives determined from some collection of infinitesimals along the lines of [4] and [8].

\section{REFERENCES}

1. Gordon E. I., Kusraev A. G., and Kutateladze S. S., Infinitesimal Analysis: Selected Topics, Kluwer Academic Publishers, Dordrecht etc., 2002.

2. Kusraev A. G. and Kutateladze S. S., Subdifferential Calculus:Theory and Applications, Nauka, M., 2007.

3. Bedelbaev F. F., Some Problems of Subdifferential Analysis and Their Applications (Kandidat Thesis), Institute of Mathematics and Mechanics of the Academy of Sciences of Kazakhstan, Almaty, 1984.

4. Rockafellar R. T., Generalized directional derivatives and subgradients of nonconvex functions, Canad. J. Math. 80 (1980), no. 2, 257-280.

5. Bonnans F., Cominetti R., and Shapiro A., Second order optimality conditions based on parabolic second order tangent sets, SIAM J. Optimization 9 (1999), no. 2, 466-492.

6. Kutateladze S. S., On a cone of a Clarke type, Optimization 35 (52) (1985), 10-15. (Russian)

7. Jimenéz B. and Novo V., Second order necessary conditions in set constrained differentiable vector optimization, Math. Meth. Oper. Res. 58 (2003), 299-317.

8. Kutateladze S. S., Epiderivatives defined by a set of infinitesimals, Sib. Math. J. 28 (1987), no. $4,628-631$.

Sobolev Institute of Mathematics

Novosibirsk 630090, RUSSIA

E-mail address: sskut@math.nsc.ru 